\title{
SOBRE LA NECESIDAD DE REPENSAR LA EDUCACIÓN DESDE EL VÍNCULO PEDAGÓGICO
}

\author{
Cristina Muñoz Morán \\ Coordinadora de la carrera de Educación Especial \\ Facultad de Ciencias Sociales \\ Universidad Evangélica de El Salvador \\ cristina.muñoz@uees.edu.sv
}

\begin{abstract}
Hablar sobre educación es algo cotidiano, pues desde diferentes medios se abordan aspectos relacionados con su importancia, con el rol que juega en la sociedad del conocimiento o su vinculación con la tecnología, entre otros temas de interés. Ahora bien, más allá de tratar de realizar un estado del arte sobre cómo se encuentra la educación en el país, se propone una breve reflexión acerca de las relaciones que se establecen actualmente en el escuela, ya que por medio de ese encuentro se produce el hecho educativo.
\end{abstract}

Entonces, al colocar la mirada en la relación pedagógica, es decir, en ese vínculo que se genera entre los diferentes actores que tienen como objetivo común el aprendizaje, es necesario conocer a ese otro con quien se construirá la relación. Además, ese conocer al otro en el contexto educativo implica tomar consciencia tanto de las creencias previas que se tienen sobre el otro como de las condiciones en que se da la relación. Pues estos elementos que están presentes antes de que se desarrolle el vínculo, pueden favorecer u obstaculizar el encuentro y, por ende, la mediación del aprendizaje.

Por tanto, se hace necesario que el docente conozca las creencias, estereotipos o experiencias previas que posee para poder generar condiciones que favorezcan un auténtico encuentro, con base en la escucha y el respeto mutuo. Y también cabe mencionar que, para que surja una relación con las características que se han mencionado, es preciso un cambio, tanto en la manera en que cada persona se ve a sí misma como en la forma de reconocer al otro. Mientras no se dé ese cambio, difícilmente se transformarán las relaciones que se establecen en la escuela, pues pasar de un vínculo despersonalizado donde prima la desconfianza, a acoger al otro a través del diálogo y la valoración de la singularidad, requiere de un compromiso personal.

Entonces, este puede ser un primer paso bastante ambicioso, pero necesario en el escenario actual, donde las redes sociales, la inmediatez o la gratificación continua van diluyendo cada vez más el valor de los encuentros. Esto con frecuencia conlleva a la invisibilización de la persona, pues en el espacio virtual su presencia se vuelve intangible y efímera. Por tanto, el desafío está en redescubrir nuestra humanidad, dejarse conmover por ésta para poder restaurar los vínculos que configuran el tejido social. 
Por otra parte, la forma en que se da la relación con el otro y el cómo se producen los vínculos entre los diferentes actores educativos son elementos a tener cuenta en la práctica pedagógica, pues las permean en los diversos momentos en que ésta se desarrolla. Así, gestos tan sencillos como la escucha, el mirar, el sentir o el estar atento al otro pueden ser el punto de partida para proponer experiencias o posibilitar nuevos encuentros en la escuela, que logren incidir en el tipo de lazos que se establecen entre docentes, estudiantes y familias. Sin embargo, cabe mencionar que en esos encuentros cada actor es responsable de cómo se ubica en la relación con el otro y de las palabras que mediarán ese encuentro, donde el docente puede diseñar diferentes experiencias, pero al final no tendrá el control sobre lo que suceda en ellas.

Por lo tanto, el docente tiene un rol clave no solo como mediador de aprendizajes, sino también como tejedor de vínculos desde los cuales puede aportar a la transformación social. Ahora bien, es momento de repensar cómo se está desarrollando la relación pedagógica en el aula o en la escuela, si se prioriza la transmisión de conocimientos o el desarrollo de habilidades que permitan producirlo, con quién se realiza el vínculo, son horizontales las relaciones que se generan... Estos son algunos planteamientos que muchas veces quedan en segundo plano en el día a día en la escuela y es necesario traerlos a escena para no correr el riesgo de mantener las desigualdades e injusticias que están tan enraizadas en nuestra sociedad.

Finalmente, tomar consciencia sobre la manera en que se reconoce al otro en el contexto escolar y cómo se generan las relaciones en dicho espacio son retos para el sistema educativo actual. Por lo que, no basta con apostarle a la innovación tecnológica, al diseño curricular, la evaluación de los aprendizajes o la formación de competencias, pues su impacto en la calidad de vida y el desarrollo de la persona es limitado sino se tienen en cuenta los vínculos pedagógicos que median dichos procesos. Además, dichos vínculos son los que dinamizan la transformación, pues el hecho educativo por sí solo no es suficiente, se necesita afectar a la persona. Así, como proponía Paulo Freire, "la educación no cambia el mundo, cambia a las personas que van a cambiar el mundo", y para ese cambio es necesario un trabajo colectivo, por lo que si no existe un vínculo que facilite la cohesión de los ciudadanos difícilmente se logrará una auténtica transformación social. 